\title{
CAD of Ion Mirror as an Aberration Corrector
}

\author{
Hussain A. Waheeb ${ }^{*}$ and Hala F. Abass ${ }^{* *}$ \\ Department of Physics, College of Science, Al-Nahrain University, Baghdad-Iraq. \\ *Corresponding Author: alhussaini83@yahoo.com. \\ Corresponding Author: hala_fadal15@yahoo.com.
}

\begin{abstract}
The present work have has been focused on suggesting a mathematical form of an axial potential distribution for an ion mirror. The equation that has been found gave us the best value of the mirror function. The Bimurzaev techniques had been investigated to find mirror trajectory using RungeKutta method. The profiles of the mirror that had been applied under Bimurzaev techniques lead us to conclude that the mirror has two electrodes. The coefficients of spherical and chromatic aberrations of the mirror have been computed and normalized in terms of the focal length. The choice of the mirror depends on the operational requirements, i.e. whether it is intended operate the electrostatic mirror in an optical system at low spherical aberration mode or at low chromatic aberration mode. [DOI: 10.22401/JNUS.20.4.07]
\end{abstract}

Keyword: Ion mirror, Spherical Aberration, Chromatics aberration.

\section{Introduction}

The idea of using an electron mirrors to correct the chromatic and spherical aberration of a axisymmetric lenses dates back more than half of century. It was found that an electrostatic mirror could correct the spherical and chromatic aberrations of the accelerating field of an immersion objective lens [1]. Unlike the usual optical light mirrors, where the reflection occurs at the physical surface, the ion or electron mirrors represents a "soft" mirror, which allows the charge particles to penetrate into nonhomogeneous reflecting medium formed by the electrostatic potential [2]. The resolution and sensitivity of the electron microscope with the mirror objective lens free of axial spherical and chromatic aberrations are higher than those of usual microscopes using electron lenses [3]. One can define the electrostatic mirror as a space-filling electrostatic field shaped by the surrounding electrodes [4]. There are several difficulties associated with the use of the mirror, including that of providing a physical separation between the entering and emerging chare particles beams [5].

Electrostatic mirror are made in various geometries according to their function in an electron optical instrument. They can be made in the form of two or three concentric cylindrical electrodes at different potentials for reducing aberration [6].
Ion mirrors are difficult to optimize and expensive to construct and Maintenance. In addition, the conventional design has two or more regions with different intensities of electric field. Electrode shapes could include (cylindrical and rectangular shapes) could also be used to fabricate effective ion mirror [7]. The field inside an electrostatic ion mirror is curved along the axis and according to the Laplace equation it also has a curvature in a radial (or transverse) direction [8]. For large distance involved between ion source and the entrance of the ion mirror it is important to position a set of weakly focusing ion optical lenses in the field free region that controlled the divergence of the beam [9]. There are many applications of electrostatic ion mirror such as TOFMS (Time-Of-Flight-MassSpectrometer) that is the most convenient way to analyze the ions mass distribution produced from ion source, and correct both spatial and the resulting energy spread of the ions originated from the source. This correcting helps to improve the resolution of image [10, 11]. For high spatial resolution and good image quality required of spectrometer system, the electrostatic ion mirrors becomes a critical part [4]. The simulation of an ion mirror gives good idea about the necessary potential distribution to solve our problem [12]. 
Trajectory equations of electrostatic mirror and spherical and chromatic aberrations coefficients

In the present research the Bimurzaev techniques have been used and modified for solving electrostatic mirror trajectory by using the paraxial ray equation of charge particle moving in an electric field. This trajectory equation have been applied twice, at the first time we applied zero magnification condition when the ay $P(z)$ represent the incident charge particle on the electrostatic mirror in equation (1) and at second time we applied the infinite magnification condition when the ray $G(z)$ represent the reflected charge particle from the electrostatic mirror in equation (2). By applying this technique we got a complete trajectory of charged particles in an electrostatic mirror system.

$\mathrm{P}(1)=1 \quad \mathrm{P}^{\prime}(1)=0$ zero magnification condition

$$
\begin{aligned}
& C_{S}=\frac{U^{-1 / 2}}{16 R^{\prime}} \int_{i}^{z_{i}}\left(\frac{5}{-}\left(\frac{U^{\prime \prime}}{U}\right)^{2}+\frac{5}{24}\left(\frac{U^{\prime}}{U}\right)^{4}+\frac{14}{3}\left(\frac{U^{\prime}}{U}\right)^{3} \frac{R^{\prime}}{R}-\frac{3}{2}\left(\frac{U^{\prime}}{U}\right)^{2} \frac{R^{\prime}}{2}\right) \sqrt{U R}{ }^{4} d z \\
& C_{C}=\frac{U^{1 / 2}}{R^{\prime 2}} \underset{z_{0}}{z_{i}}\left(\frac{U^{\prime}}{2 U} R^{\prime} R+\frac{U^{\prime \prime}}{4 U} R^{2}\right) U^{-1 / 2} d z
\end{aligned}
$$

Where, $\mathrm{C}_{\mathrm{s}}$ and $\mathrm{C}_{\mathrm{c}}$ are the coefficients of spherical and chromatic aberrations respectively, $\mathrm{R}$ is the radial displacement of the beam in the electrostatic mirror system. The integration given in the above equations are executed by Simpson's rule.

\section{Results and Discussion:}

The suggested axial potential for the electrostatic ion mirror are given in equation (3) represents the suggested axial potential model that has been modified to achieve an electrostatic mirror with accelerating mode and eliminated spherical and chromatic aberrations.

$$
U(z)=a * \tanh \left(\frac{z}{c}\right)+b * \cosh \left(\frac{z}{c}\right)
$$

Where, $\mathbf{a}, \mathbf{b}$ and $\mathbf{c}$ are the parameters where $\mathrm{a}, \mathrm{b}$ in volt and $\mathrm{c}$ in millimeter. From the Fig.(1) we can see the axial potential distribution at the mirror length with first and $\frac{d^{2} P}{d z^{2}}+\frac{U^{\prime}}{2 U} \frac{d P}{d z}+\frac{U^{\prime \prime}}{4 U} P=0$

$\mathrm{G}(\infty)=0 \quad \mathrm{G}^{\prime}(\infty)=1 \quad$ infinite magnification condition

$\frac{d^{2} G}{d z^{2}}+\frac{U^{\prime}}{2 U} \frac{d G}{d z}+\frac{U^{\prime \prime}}{4 U} G=0$

Here, $P^{\prime}=\frac{d P}{d z}$ and $G^{\prime}=\frac{d G}{d z}$ are the first derivatives of radial displacement of incident and reflected paraxial ray from the electrostatic mirror respectively, $\mathrm{z}$ represent the optical axis, and $U(z)$ is the axial potential distribution.

The spherical and chromatic aberrations coefficients can be calculated by using the following formula: second derivatives of the potential. $\mathrm{U}(\mathrm{z})$ are the axial potential distribution suggested.

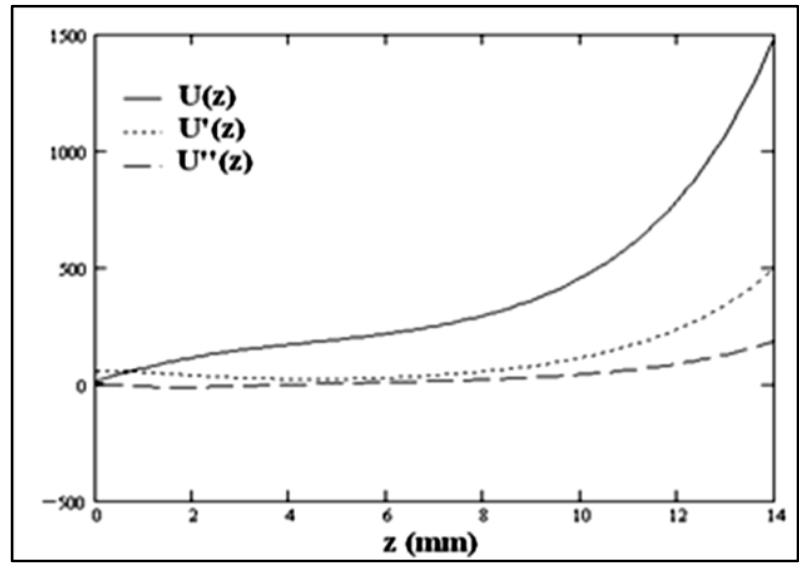

Fig.(1): The axial potential distribution $U(z)$, first and second derivatives $U^{\prime}(z)$ and $U^{\prime \prime}(z)$ as a function to the length of electrostatic mirror when $a=150, b=15, c=2.7$, and mirror length $L=14 \mathrm{~mm}$. 
Fig.(2) show the ion beam paths along the electrostatic mirror field at different values of parameter $\mathbf{b}$ where the most favorable values of the mirror is deduced having the following parameters $\mathbf{a}=150$ volt, $\mathbf{b}=15$ volt and $\mathbf{c}=2.7$ $\mathrm{mm}$ at the mirror length $\mathbf{L}=14 \mathrm{~mm}$.

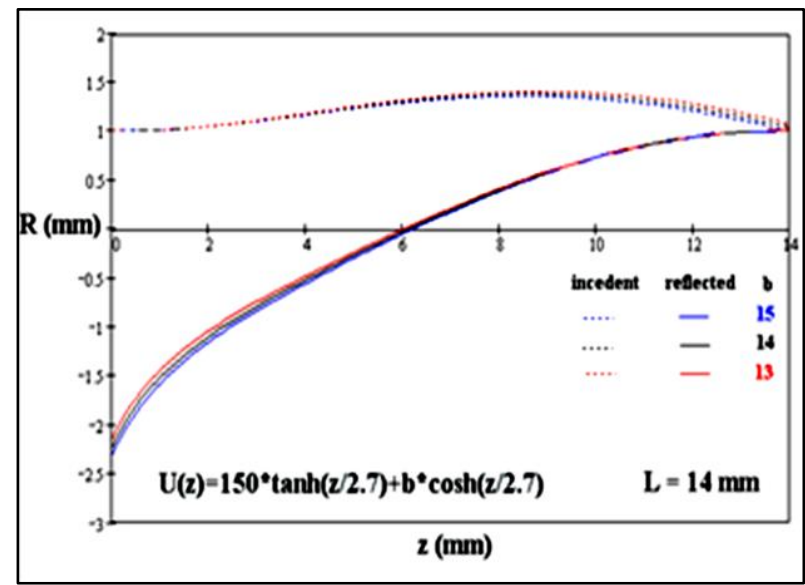

Fig.(2): The ion beam trajectory for the electrostatic mirror under various values of $b$ when $a=150$ volt, $c=2.7 \mathrm{~mm}$, and mirror length $\mathrm{L}=14 \mathrm{~mm}$.

From the calculation we deduced that the increasing values of $\mathbf{b}$ directly proportional with electrostatic mirror focal length but the increases value of parameter a cause to decrease the electrostatic mirror focal length. Figs. (3) and (4) show the changeability of spherical and chromatic aberrations coefficients normalized to the mirror focal length and both taken as a function of the mirror length normalized to the mirror focal length L/f at different values of parameters a and $\mathbf{b}$.

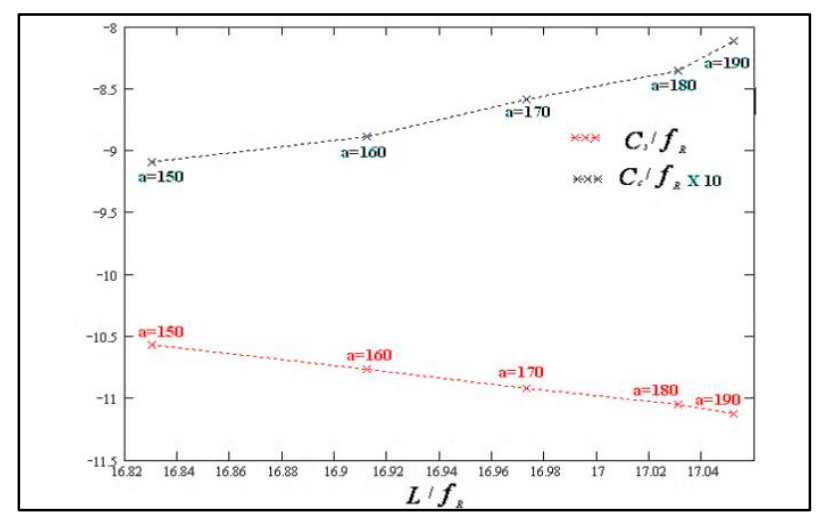

Fig.(3): Show the changeability between $C_{s} / f_{R}$ and $C_{c} / f_{R}$ as a function of relative electrostatic mirror length $L_{/} f_{R}$ at $b=15$ volt, $L=14 \mathrm{~mm}$, and $c=2.7 \mathrm{~mm}$.

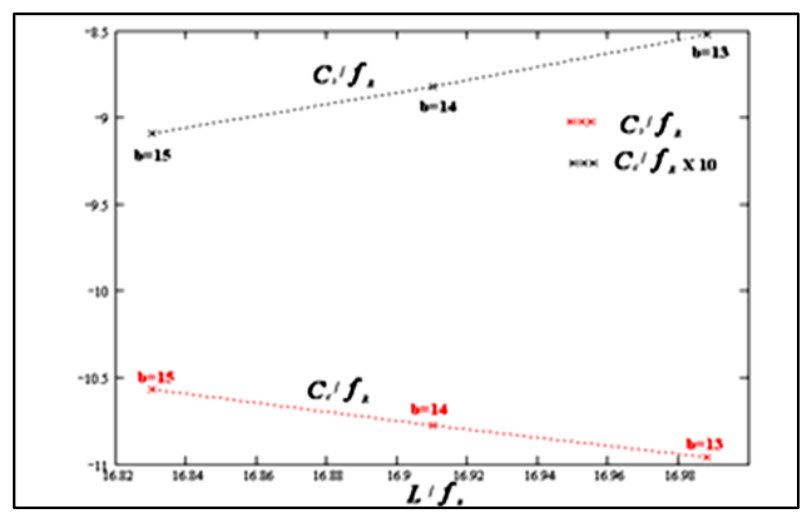

Fig.(4): Show the changeability between $C_{s}{ }^{\prime} f_{R}$ and $C_{c}{ }^{\prime} f_{R}$ as a function of relative electrostatic mirror length $L_{L} / f_{R}$ for different values of $b$ at $a=150$ volt, $L=14 \mathrm{~mm}$, and $c=2.7 \mathrm{~mm}$.

It is noted from Fig.(3) that the increasing values of the parameter a causes an increases in both of $C_{c} / f_{R}, L / f_{R}$ and decreases of the $C_{s} / f_{R}$ with the keeping $\mathbf{b}=15$ volt for different mirror lengths with specific constant c. From Fig.(4) we can see that the increasing values of b causes to increase the values of $C_{s} / f_{R}$ while the values of $C_{c} / f_{R}$ and $L / f_{R}$ are decreasing.

Fig.(5) show the profile of the ion mirror electrodes as a function of optical axis normalized to the mirror length at most favorable parameters where $\mathbf{a}=150$ volt, $\mathbf{b}=15$ volt and $\mathbf{c}=2.7 \mathrm{~mm}$ at mirror length $\mathbf{L}=14 \mathrm{~mm}$. The distance between two electrodes is $0.03 \mathrm{z} / \mathrm{L}$.

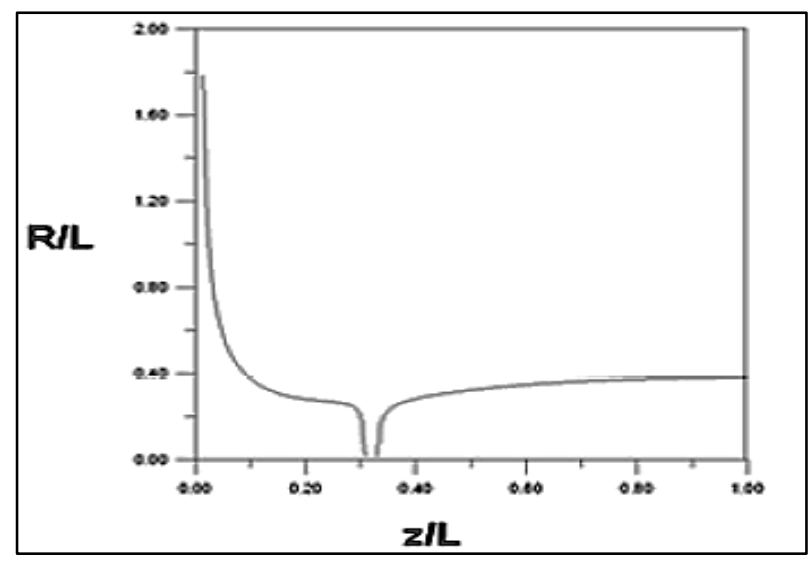

Fig.(5): The electrodes profile of an electrostatic mirror when the parameters $a=150$ volt, $b=15$ volt and $c=2.7 \mathrm{~mm}$ at mirror length $\mathrm{L}=14 \mathrm{~mm}$. 


\section{Conclusion}

The concepts that had been suggested in this research with regard to the mathematical expressions for the axial potential and the ion beam to calculate the aberrations for ion mirror are successful and gave rise to excellent result from the ion-optical point of view. We can see from our results that the spherical aberration coefficient $\left(\mathrm{C}_{\mathrm{s}}\right)$ increasing when the parameter (b) is decreasing. The Chromatic aberration coefficient $\left(\mathrm{C}_{\mathrm{c}}\right)$ decreases as the parameter (b) decrease. And the spherical aberration coefficient $\left(\mathrm{C}_{\mathrm{s}}\right)$ decreases as the parameter (a) increase. The Chromatic aberration coefficient $\left(\mathrm{C}_{\mathrm{c}}\right)$ increases as the parameter (a) increase. The negative values of the aberration coefficient give rise to the possibility of using these devices to eliminate the aberration. Two electrodes of electrostatic ion mirror has been achieved which can be useful for application in studying a specimen surface.

\section{References}

[1] Schmid, P., Feng, J., Padmore, H., Robin, D., Rose, H., Schlueter, R., Wan, W., Forest, É., and Wan, Y., "Correction and alignment strategies for the beam separator of the photoemission electron microscope" (PEEM 3), Rev. Sci. Instrum., 76, 1-14، 2005

[2] Rose, H., and Wan, W. (), "Aberration correction in electron microscopy", Proc. IEEE, Particle Accelerator Conference, Knoxville, Tennessee, 44-48, 2005.

[3] Bimurzaev, S. B., Serikbaeva, G. S., and Yakushev, E. M., "Electrostatic mirror objective with eliminated spherical and axial chromatic aberrations", J. Electron Microsc., 52, 365-368, 2003.

[4] Henkelman, R. M., and Ottensmeyer, F. P., "An electrostatic mirror", J. Phys. E: Sci. Instrum., 7, 176-178, 1973.

[5] Crewe, A. V., Ruan, S., Korda, P., and Tsai, F. C., "Studies of a magnetically focused electrostatic mirror, I. Experimental test of the first order properties", J. Microscopy., 197, 110-117, 2000.

[6] Berger, C., and Baril, M., "Studies of three-cylinder electrostatic mirrors and lenses”, J. Appl. Phys., 53, 3950-3956, 1982.

[7] Zhang, J., Gardner, B. D., and Enke, C. G., "Simple geometry griddles ion mirror", J. Am. Soc. Mass Spectrum., 11, 765-769, 2000.

[8] Doroshenko, V. M., and Cotter, R. J., "Ideal velocity focusing in a reflection time-of-flight mass spectrometer", J. Am. Soc. Mass Spectrom., 10, 992-999, 1999.

[9] Giannakopulos, A. E., Thomas, B., Colburn, A. W., Reynolds, D. J., Raptakis, E. N., Makarov, A. A., and Derrick, P. J., "Tandem time-of-flight mass spectrometer (TOF-TOF) with a quadratic-field ion mirror", Rev. Sci. Instrum., 73, 2115-2123, 2002.

[10] Vialle, J. L., Baguenard, B., Bourgey, A., Cottancin, E., Lermé, J., Palpant, B., Pellarin, M., Valadier, F., and Broyer, M., "A cylindrical reflectron time-of-flight mass spectrometer", Rev. Sci. Instrum., 68, 2312-2318, 1997.

[11] Chernushevich, I. V., Loboda, A. V., and Thomson, B. A., "An introduction to quadrupole-time-of-flight mass spectrometry", J. Mass Spectrom., 36, 849865, 2001.

[12] Hohl, M., Wurz, P., Scherer, S., Altwegg, K., Balsiger, H., "Mass selective blanking in a compact multiple reflection time-offlight mass spectrometer", International J. Mass spectrometry, 188, 189-197, 1999. 